\title{
Energy Efficient Camera Solution for Video Surveillance
}

\author{
Misbah Ahmad ${ }^{1}$, Imran Ahmed ${ }^{2}$, Kaleem Ullah ${ }^{3}$, Iqbal Khan $^{4}$, Ayesha Khattak ${ }^{5}$, Awais Adnan ${ }^{6}$ \\ Center of Excellence in Information Technology \\ Institute of Management Sciences (IMSciences) Hayatabad, Peshawar (Pakistan)
}

\begin{abstract}
Video surveillance is growing rapidly, new problems and issues are also coming into view which needs serious and urgent attention. Video surveillance system requires a beneficial energy efficient camera solution. In this paper, a single overhead camera solution is introduced which overcomes the problems existing in various frontal and overhead based surveillance systems. This will increases the efficiency and accuracy of surveillance system i.e. frontal and overhead. In this paper, two energy efficient overhead camera models are presented. The first model consists of a single overhead camera with a wide angle lens which covers a wide field of view addresses problems present in the traditional surveillance system. The second model, presents a single smart centralized overhead camera which controls various frontal based cameras. Several factors associated with camera models such as field of view, focal length and distortion are also discussed. Impact of the surveillance cameras are finally discussed which shows that a single energy efficient overhead camera surveillance system can solves many problems present in traditional surveillance system like power consumption, storage, time, human resource and installation cost and small coverage area.
\end{abstract}

Keywords-Energy efficient; video surveillance; overhead camera

\section{INTRODUCTION}

Nowadays in computer vision, one of the active, attractive and most important research area in video surveillance. The estimated urban population in 2030 will be round about 5 billion which is $60 \%$ of the total world population [1]. With this significant increase in the population, people might suffer from a series of security and privacy threats. Various government organizations are spending billions of dollars every year on domestic security around the globe. The sole purpose of spending this huge amounts of the money is to enhance the surveillance systems. Further more, with increasing trend of camera installation at both public and private places, a huge amount of data and storage are also required.

The process of monitoring certain objects/scene called surveillance. There are basically three types of video surveillance i.e manual, semi-autonomous and fully-autonomous. One of the widely used video surveillance type is manual, where a video content is analyzed by a human operator. While Semiautonomous video surveillance required significant human intervention along with video processing (e.g simple motion detection). In contrast the fully-autonomous video surveillance system, has no human intervention at all. The input video sequence is taken and high-level decision-making tasks are performed including abnormal event detection and gesture recognition [2]. With an increase in urbanization, a rising economy and social transformation people have started moving from rural areas to cities, which results in further increase of cities population.

Information technology has a significant impact on human life. Information and communication are highly deployed resource in our daily lives. For security purposes, various surveillance cameras (including sensors, smart cameras) are installed all over the place in the cities (e.g banks, shopping malls, airports and streets etc). These installed cameras capture a large number of videos and images which are used for various surveillance purposes. Some of these videos may also pose a threat to the privacy of people. These surveillance systems require human operators for constant monitoring which means that the effectiveness and response of these surveillance systems are highly dependent on fully vigilant human monitoring capabilities. Furthermore, the area under surveillance and the total number of cameras installed at a particular location is also limited by the human resource availability. The long duration of video monitoring by the human operator is also impractical and unfeasible. Some of under develop countries also suffering from energy crises (electricity), therefore in such circumstances continuous supply of electricity to surveillance system is difficult. So, in this paper, we present an energy efficient single camera solution that can be operated on less amount of power requirement by a small number of human operators.

Single Overhead Camera plays a vital role in overcoming the problems present in both traditional frontal and overhead based surveillance system. In this paper, an energy efficient camera solution is presented which helps to overcome certain issues like camera installation cost, power consumption, human resource, occlusion handling and privacy. Energy Efficient camera solution has gained a lot of attention in the recent years in many applications, not limited to traditional surveillance system, but includes developing fields such as elderly care, entertainment or home automation. The developed idea is energy efficient and affordable solution which improves, security and video surveillance. The single overhead based camera solution can benefit people and the city in a variety of aspects: energy, environment, industry, living, and services.

The main contribution of this work is to propose an energy efficient camera model, which can be further expended to control other surveillance cameras. Furthermore it is discussed, how this model can improve the existing surveillance system and how it can be applied in the privacy protection system. Lastly, the impact and factors of the developed energy efficient overhead based camera model on the surveillance system is also discussed. The remainder of the paper is organized as follows: In Section II we summarize the related work, in 
Section III we presented the developed camera models while in Section IV we discuss different factors and impacts of presented models while Section V concludes the paper.

\section{RELATED WORK}

In video surveillance a variety of work has been done in last few decades based on frontal based camera system. Mikolajczyk et al. [3] described the method for detecting human in a single image which can detect full body as well as close up views in presence of clutter and occlusion. MIT pedestrian data set is used for training and testing. Yeh et al. [4] proposed a dual cooperative camera system for frontal surveillance (Fig. 1). Two cameras have been used correctly tracking the target object. The two cooperative cameras were able to correctly analyze the human body shape while the other predict the motion, position and height of human body. The experimental results shows that proposed algorithm performed well considering clear close up views for both multiple and single object.

Natarajan et al. [5] highlighted several issues in existing frontal based surveillance systems. In this survey they focused on Multi-Camera Coordination and Control (MC3) architectures and the functionalities for both centralized and distributed camera architecture. Bialkowski et al. [6] developed a person re-identification method based on color and texture models. A new challenging multicamera surveillance database was developed. They captured indoor video sequence with people in different poses. Chua et al. [7] studied out the unusual behaviour of a person in surveillance video sequence. They presented vision-based fall detection technique for human. Due to the property of human shape that varies from different camera angles instead of conventional ellipse or bounding box techniques. They focused on three points to represent a person shape. The technique is based on human shape. Cohen et al. [8] proposed an overhead based person recognition system. They considered top view images of the persons. Therefore Dataset of 12 individuals (Low quality images) each having 60 frames of multiple poses like sitting, standing, different rotations and translations were used. A Smart conference room (constrained environment) was considered for this purpose where cameras were mounted on the ceiling so that it cover the entire room. Ahmed et al. [9] proposed a new feature based algorithm for detecting person from extreme position by using an overhead camera in industrial environment. Three type of appearance viewed were observed i.e. direct under the camera, the diagonal view and when person moves away from camera. SCOVIS, a real-world industrial dataset was used. Another overhead based person detection work has also been done by [10].

Nakatani et al. [12] proposed an image based person identification method from top-View. The person's area in a captured image was identified first by using background subtraction. The proposed method was valid only in the situation where people stop in front of a door. Pizzo et al. [13] presented a method for counting people using zenithal mounted cameras which were able to provide accurate counting under different realistic conditions. Real world environments were adopted where people counting was characterized by natural illumination (OUT-DOOR) or where the source of illumination was very artificial (INDOOR). Garca et al. [14] used a single fixed camera for detecting people from overhead view and tried
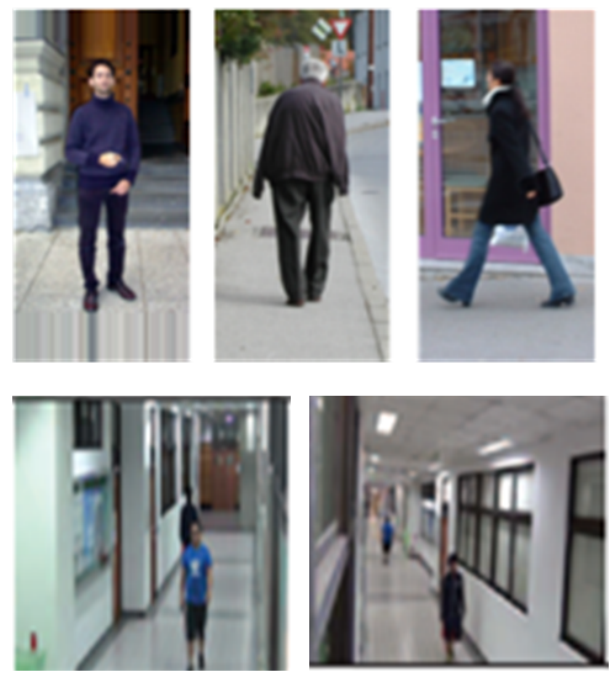

Fig. 1. Some of the images captured from frontal surveillance cameras. [4], [11]

to solve the problem of occlusion. An efficient and reliable feature descriptor for human detection in a top-view depth image was presented by [15] (see Fig. 2).

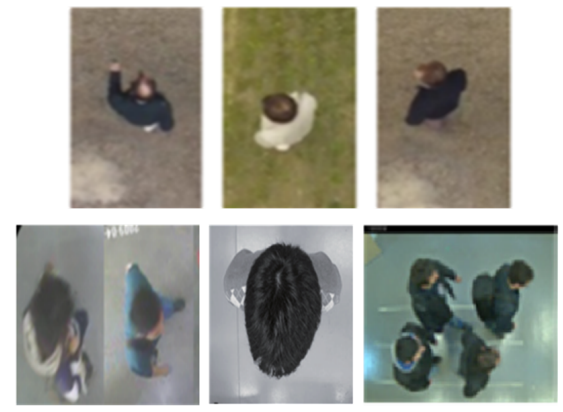

Fig. 2. Some of the images captured from Overhead survillance cameras [16] [17] [18]

Carletti et al. [19], presented a top view method based on depth map images for person detection. The depth images were captured using single sensor mounted in zenithal position in both indoor and outdoor environments. SIFT-FAST algorithm for counting people in a crowded environment was proposed by [20]. They used traditional CCTV video sequences for detection purposes. To address the variation in human visual appearance Paul Blondel et al. [16] considered a multi-view camera images. The Multiview images were captured from both frontal, azimuthal and overhead view. Liu et al. [21] proposed a new hybrid-overlapping alliance of cameras for the purpose of tracking both overlapping and non-overlapping. They focused on depth images instead of RGB. Burbano et al. [22] focused on embedded smart camera network to detect and track people from overhead view. Tseng [23] proposed a realtime surveillance system comprised of multiple depth cameras located indoor for person detection and tracking.

\section{Conceptual Camera Models}

In this section, we presented an overhead based single energy efficient camera models. These models are able to 
perform well in several real-life scenarios as discussed in the introduction. It also helps to overcome the occlusion problems, in traditional frontal surveillance system, as can be shown in Fig. 3. In the first row of Fig. 3 it can be clearly seen that in different real-life environments the camera recording contained different occlusion problems either by some other object (heavy machinery, walls, or other person) or sometimes due to self-occlusion. The overhead camera can overcome the occlusion problem as seen in the second row of Fig. 3 in much better way. It can be seen that single overhead camera easily overcome the problem of occlusion in such scenarios e.g person occluded by each other or by some heavy machinery. In this section different camera parameters including field of View $(F O V)$, focal length of lens and distortion effect are also discussed briefly. Fig. 3 shows that in most of the cases the traditional frontal based camera surveillance system suffers from occlusion problem.
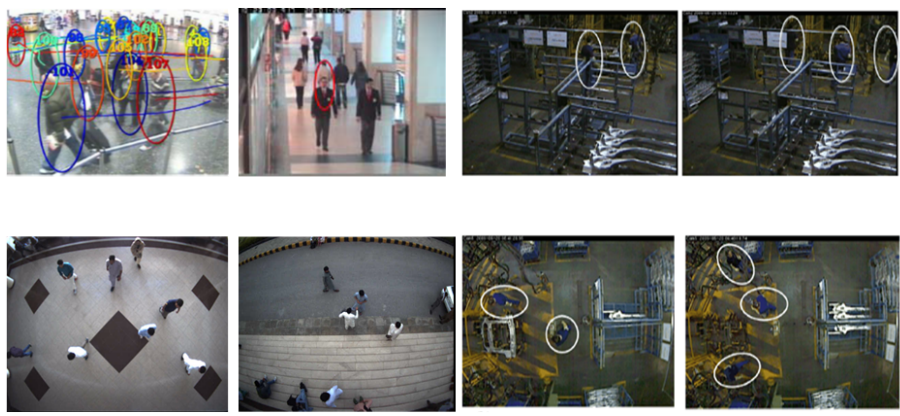

Fig. 3. In first Row images were captured by frontal camera that shows the issue of occlusion while in second row some of the images are shown that were captured by single overhead camera that solves the issue of occlusion. [10] [24]

\section{A. Single Overhead Energy Efficient Camera Model}

This paper introduce energy efficient single overhead camera solution that saves the power consumption issues of the surveillance system. The Single Overhead Based Energy Efficient Camera Model covers wide scene as shown in Fig.4 and solves the problem of occlusion. The Field of view $(F O V)$ for the overhead camera has also been calculated as shown in Fig.4. Different Camera parameters including Focal Length and distortion caused by camera are also discussed in this section.

1) Field of View vs. Height: The field of view of overhead camera has been shown in below Fig. 4. It can be clearly seen in the image that $F O V$ depends on camera installation height $h$, width $w$ and length $l$ of the scene. To calculate the field of view from the Fig. 4 the below equation can be considered.

$$
F O V=2 \tan ^{-1} \frac{w}{h}
$$

In equation $1 h$ is the height of camera from floor and $w$ is the width of scene. The $F O V$ is represented as $\theta$. So that equation 1 can also be written as:

$$
\theta=2 \tan ^{-1} \frac{w}{h}
$$

From Fig. $4 \theta$ can be calculated as:

$$
\theta=2 \alpha
$$

The equation 3 can be also be written as follows:

$$
\alpha=\frac{\theta}{2}
$$

By putting the value of $\theta$ in equation 4 we get;

$$
\alpha=\tan ^{-1} \frac{w}{h}
$$

In case where $\theta$ or $\alpha$ and width $w$ is given and camera height $h$ is unknown, using equation 5 camera height can be calculated as:

$$
h=\frac{w}{\tan \alpha}
$$

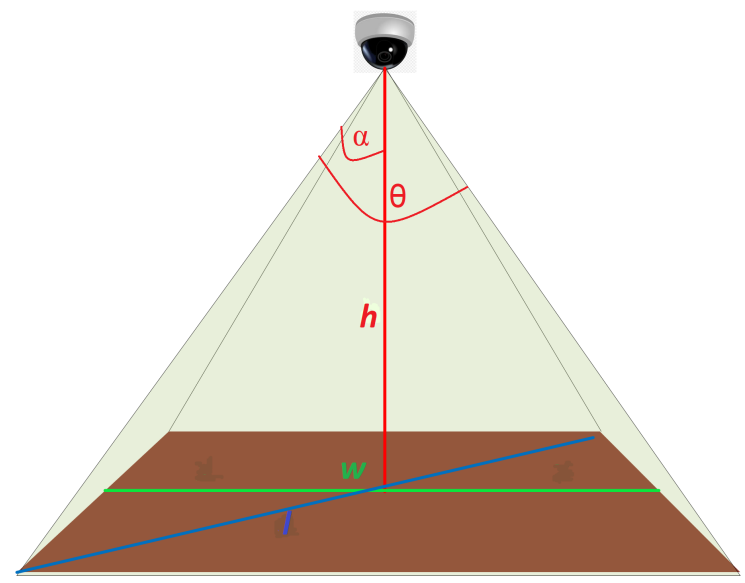

Fig. 4. The FOV of the overhead camera: $h$ represents height, $w$ represents width, $l$ is the diagonal length of scene. While $\theta$ is the field of view FOV

2) Using Focal Length to calculate FOV: The coverage area or $F O V$ of overhead camera can also be calculated using focal length of the lens. Basically focal length is the distance between the lens and the image sensor when the subject is in focus, usually camera comes with focal length (e.g. $28 \mathrm{~mm}$, $50 \mathrm{~mm}$, or $100 \mathrm{~mm})$. In Fig. 5 the focal length of the sensor has been shown Fig. 5 .

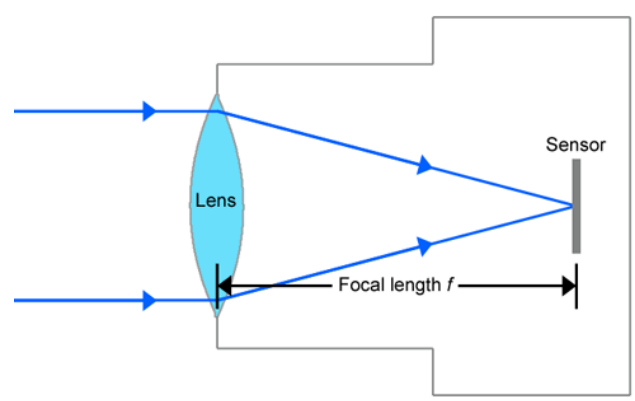

Fig. 5. Focal length of camera sensor.

To calculate the focal length of the installed camera Fig. 6 has been considered. In Fig. $6 h_{s}$ is the horizontal sensor dimension (number of horizontal pixels multiplied by the pixel 
size) $h$ is the height between installed camera position and floor while $w$ is the width of the scene.

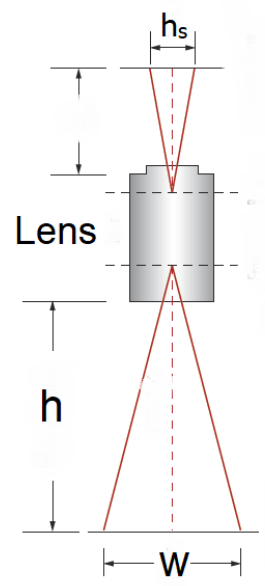

Fig. 6. The focal length of camera lens: $h_{s}$ is the horizontal sensor dimension, $h$ is the height between installed camera position and floor while $w$ is the width of the scene.

The below equation 7 is calculated using above Fig. 6. In equation $7 h_{s}$ is the horizontal sensor dimension (number of horizontal pixels multiplied by the pixel size) and $f$ is the focal length of the lens, both in millimeters; the $w$ and $h$ must be measured in the same unit system.

$$
f=\frac{h_{s} \times h}{w}
$$

By using the focal length information $F O V^{\prime}$ can also been calculated as shown in equation:

$$
F O V^{\prime}=2 \tan ^{-1} \frac{h}{2 f}
$$

where $h$ is camera height and $f$ is the focal length of camera.

In Fig. 4 it can be clearly seen that installed overhead camera provides larger $F O V$ as compared to the traditional frontal based camera. The camera installation height can be adjusted according to the application requirement. In Fig. 7 the wide $F O V$ of overhead images can be clearly seen. The overhead camera also helps to minimizes the number of installed cameras at any location which needs wider view by adjusting camera installation height. As because the wide $F O V$, it covers the entire scene as shown in below Fig. 7. In Fig. 7 it can be clearly seen that how single overhead camera is used to to cover the wide scene, which is covered by multiple frontal cameras as shown in Fig. 7.

\section{B. Hybrid Camera Model Surveillance}

This work is not limited up to just single camera model, but extends the conceptual solution to control multiple surveillance cameras. The one energy efficient overhead camera can be used as a centralized camera to handle surveillance in complex environments where we need frontal images i.e facial detection and recognition.As shown in Fig. 3, using overhead significantly solves the problem of occlusion, but at cost of two limitations i.e self occlusion (e.g. at middle of the image person torso
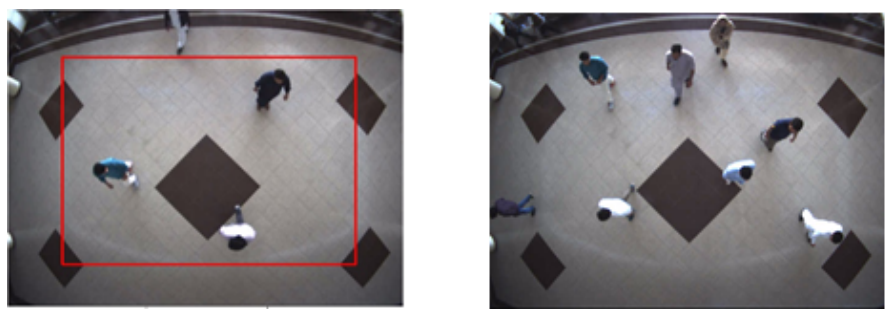

Fig. 7. Field of View of Single Overhead Camera. It can be seen that using single overhead camera at certain height provide better coverage of the wide scene.

is occluded with upper body part), another limitation of the single overhead camera model could be the clear visibility of facial images. While viewing a person from overhead view it could be difficult to capture frontal images.

To overcome these types of problems in a typical surveillance system a method is presented using a centralized camera which is further connected to others frontal cameras. So in that way where facial images or full person body is required the overhead camera enables the frontal camera to captures the video or images of the person.

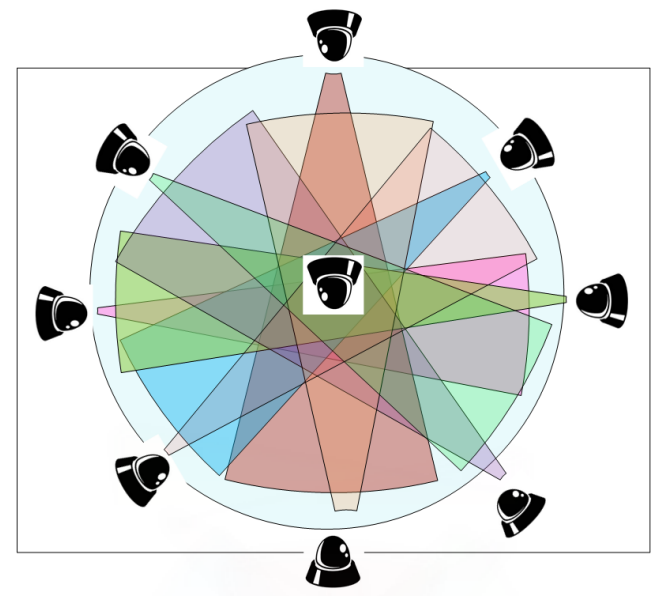

Fig. 8. Single Centralized Overhead conceptual camera Solution.

In Fig. 8 the conceptual solution of the Single Overhead Energy Efficient Camera Model is shown. It can be seen from the Fig. 8 that to cover the wide scene, eight different frontal based cameras are used while that scene can be easily cover by the one overhead camera. But in such a scenario, where the frontal images is necessary the extended conceptual model can be used to control other surveillance cameras. Like in Fig. 9 it can be seen that from F1 to F8 the frontal based cameras are installed while one overhead camera $\mathrm{O}$ is installed at center on the top of floor to control other cameras. When there is no human traffic all eight cameras will be at stand-by (not recording) mode. The particular camera will be activated by the overhead camera in case of motion at the particular location. In case if the person is coming towards F1 the overhead camera sends signal to F1 to switch on recording mode from the reset mode and start recording image or video at particular time.

Similarly if the person is moving towards F8 the overhead camera send back signal to F8 to captured the video at 


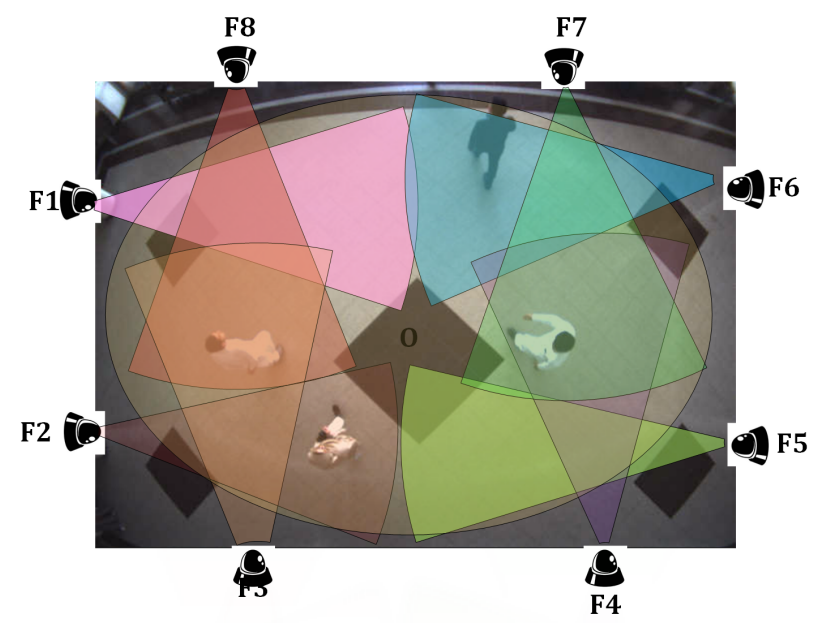

Fig. 9. Conceptual model of Single topview camera to control other frontal based. This Figure shows the conceptual model with other frontal based cameras. The $F O V$ of eight different frontal cameras has been shown by shaded area which is small as compared to Overhead Camera $O$. It can be seen that how single overhead camera covers the wide scene which is covered by multiple frontal cameras. The topview camera can also been used with multiple frontal cameras.

that movement. In such a way without effecting the power, storage and time the overhead camera enables the frontal based cameras to captured the videos when needed. In Fig. 9, one centralized camera is use as a central camera so the other cameras will be at rest or stand by the state when not needed. Now this overhead camera model helps in facial expression recognition and suspicious activity controlling scenarios etc. To control other frontal based cameras we can enhance the model using sensors that detect motion or spatial information. This also helps to save power or energy during timings where person facial recognition is not necessary.

\section{FACtors EFfecting Conceptual CAMERA MODELS}

To analyze the efficiency and effectiveness of the single overhead camera. The proposed solution provides an energy efficient camera solution for surveillance system, that can be operated on low power. So instead of reducing the resolution power of surveillance cameras, we can use single camera and record some good videos. In this section different factors have been analyzed e.g distortion, power consumption, storage, privacy issues, Human resource requirement and installation costs.

\section{A. Distortion in Overhead Images}

Overhead images provide a larger field of view FOV and solves the occlusion problems but it may suffer from image distortion. Moreover it might create problems for researchers to detect, count and track the person in overhead images accurately due to distortion. There are two types of distortions: optical and perspective. Both types result in some kind of deformation in images. Optical distortion is usually caused by the design of optical lens. Perspective distortion is due to the position of the camera relative to the subject. The overhead camera usually suffers from radial distortion as shown in Fig.
10. It can be seen, that straight lines in the left image and straight footpath edges in right image of Fig. 10 becomes radial due to the distortion.
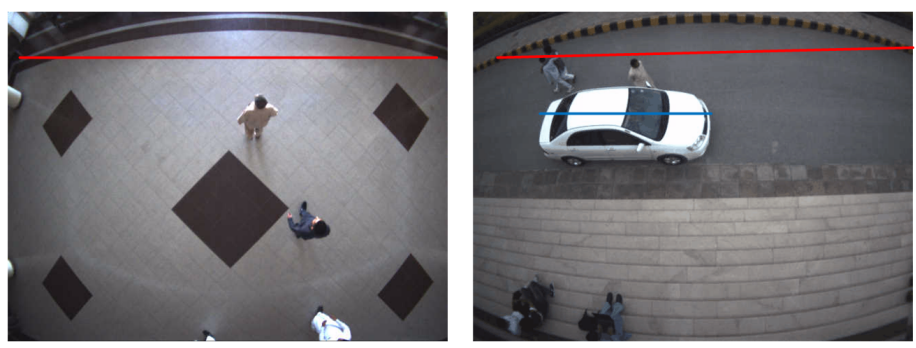

Fig. 10. Effect of distortion caused by wide angle overhead camera: The curve line at the top of both images can be seen due to radial distortion, which need to be straight.

To calculate the radial distortion for the above images. The parameters including $x_{d}, y_{d}$ and $r_{d}$ have been considered as shown in Fig. $11 r_{d}$. In Fig. $11 r_{d}$ is the radial distance, $x_{d}$ is horizontal distance and $y_{d}$ is the vertical distance of the scene. It can be seen that the distortion effect in the image increases with increase in radial distance $r_{d}$. Due to the distortion effect square boxes in Fig. 11 looks like affine rectangles.

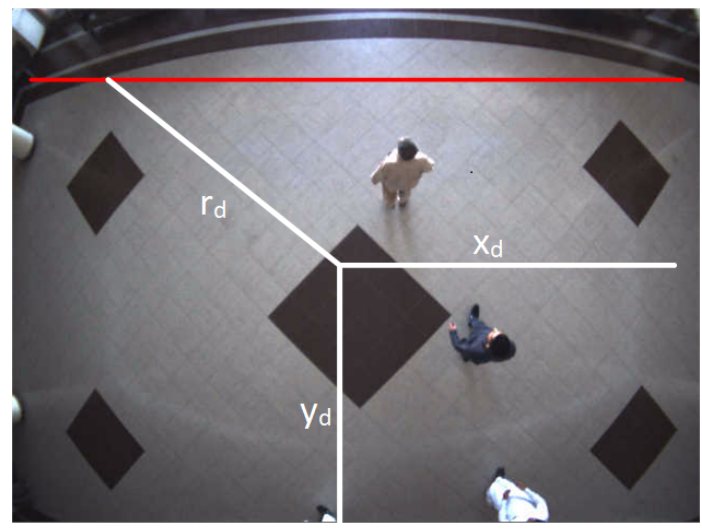

Fig. 11. Effect Radial distortion caused by overhead camera. The effect of distortion increases with increase in radial distance $r_{d}$.

The general formulae for calculating radial distortion using Fig. 11 is as follows. In equation $9 r_{d}$ is radial distance of distorted image from central points $x_{d}$ and $y_{d} . k$ is the distortion factor. By using the below equation the distorted image can be converted in to undistorted image.

$$
r_{u}=r_{d}\left(1+k_{d}^{2}\right)
$$

\section{B. Privacy Issues}

With increasing use of surveillance technology, it somewhere reducing the privacy of human. The concept of surveillance privacy varies with cultures. In most of scenarios there is no need to monitor the person from their facial images or videos so in such cases, the overhead would be the better choice to monitor the public places just from an overhead view without violating the privacy of the people.

\section{Power Consumption's}

With the increasing rate demand and supply of video surveillance more cameras and sensors are required. These 
installed surveillance cameras and sensors use a noticeable amount of power over a years' time i.e. energy for power, transmission and distribution of video or images data. According to [25] a single surveillance camera used up to 40 Watt of power per year. As a surveillance system is made up of multiple cameras or sensors, a recording device monitors and wires, etc. These energy consumption lead us to provide an energy efficient camera solution that overcomes these energy consumption's issues and provide some smart energy surveillance solution that can save energy consumption in many aspects and also prevent a blackout of power and failure of cameras and sensors. In Fig. 12 we have shown increase in the number of cameras increases the energy consumption rate in the surveillance system. As from the above discussion we have seen that using one overhead camera saves cost and energy consumptions.

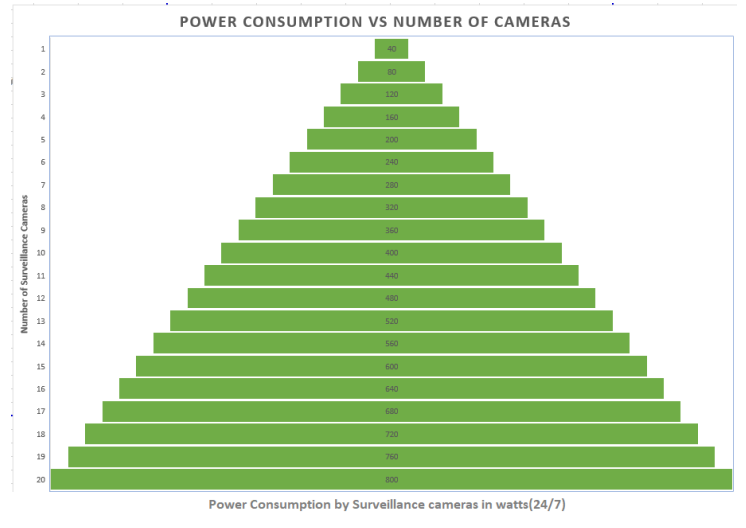

Fig. 12. The General Overview of Power Consumption per day increases with increase with the number of installed camera.

\section{Data Storage}

As discussed earlier in surveillance system with multiple cameras requires a large amount of storage space for recording videos and images. A single camera recording can occupy 25 to $50 \mathrm{~GB}$ of storage space per day. This storage space increases with increasing rate of video quality, camera stream, camera resolution, average frame rate, number of camera, per camera frame rate, per day recordings, number of desired days for storage and bandwidth etc. Similarly, the compression rate required to support the centralized storage, analysis and retrieval of an organization's growing mountain of video footage as shown in Fig. 13. After the power consumption, the second challenge is the storage, the latest HD model cameras occupied the space $25 \mathrm{~Gb}$ per day while the latest IP based camera used to HD cameras 10- $12 \mathrm{~GB}$ day (for the 2-megapixel camera). The storage capacity occupied by the surveillance cameras depends on the quality of the video. The storage capacity depends on video frame rate, resolution rate and compression rate. It also depends on the recorded video colour model which may be RGB, grey and Infrared. In Fig. 13 it can be clearly seen that how the storage increase with number of installing cameras.

\section{E. Human Monitoring}

Installing cameras and sensors are not enough for the video surveillance system. After installation, the videos are also

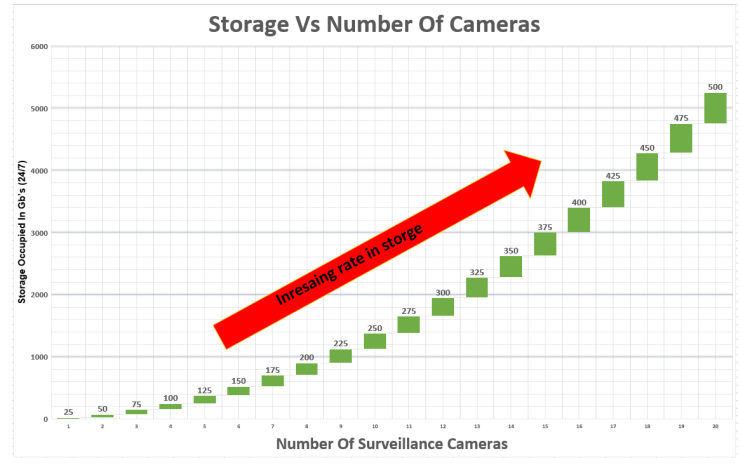

Fig. 13. Storage capacity in Gb's per day occupied by Surveillance cameras.

required to be monitored by human operators in vigilant way. Fig. 14 shows that the multiple camera footage which need proper and accurate monitoring. And as there is not a single camera in traditional surveillance system so that to properly monitor the number of cameras is also a hectic task.

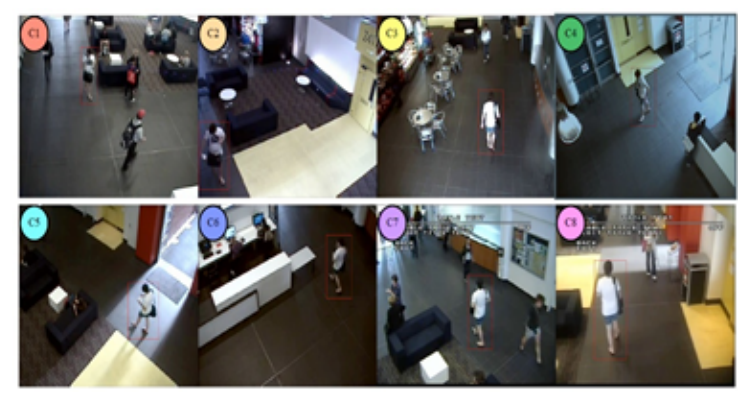

Fig. 14. Footage of multiple surveillance cameras shows the difficulty of surveillance monitoring [6].

Also, this kind of monitoring is sometime restricted to a small area because of complex environments. Some times because there is a only single human operator who is responsible for monitoring of multiple cameras. The careless of the human operator may lead to security threats. Also instead of hiring multiple human operators for the surveillance system to monitor multiple cameras by installing one overhead camera, we can saves the human resource cost too.

\section{F. Installation of Multiple Cameraś}

As with increasing rate of video surveillance instead of hiring multiple security officers for monitoring. Many organization prefer to install security cameras and sensors. Installation of multiple cameras and sensor is not easy, therefore instead of installing multiple cameras in this paper, we have presented a one camera solution that can saves the installation hectic and cost of multiple cameras by covering the wide scenes using overhead camera.

\section{G. Overall Installation Cost}

Now a days, technology able to capture of sharper and crisper IP video surveillance footages. But, such advanced technology comes at a cost particularly in terms of the investment in video surveillance storage. Apart from monitoring and power consumption, installation of multiple cameras is 
also expensive. Sometimes it is very expensive for small organization to purchase cameras and other equipment needed for the surveillance system. It is not just a one-time expense but with the advancement in technology, it is the necessary need to keep upgrading it with time. In such scenarios installing a single overhead camera is affordable and effective solution. Camera installation also depends on coverage area, larger the coverage area more number of cameras are needed, security Needs (in high security or sensitive areas), External or Internal (for outdoor you need expensive water and weather proff cameras) Wired or Wireless and Monitored (including IP based cameras) Monitored or Monitored (is the human operator required for proper monitoring or not). As shown in Fig. 15 that how the installation cost of camera installation increases with number of installed cameras.

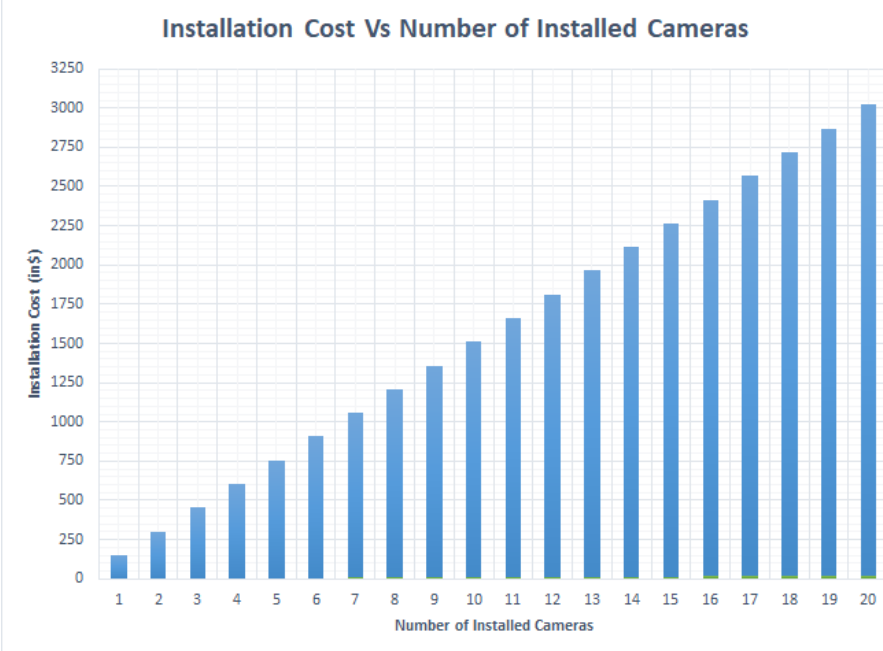

Fig. 15. The installation cost increases exponentially with increasing number of cameras.

\section{CONCLUSION}

In this paper, we discussed the different issues present in traditional surveillance systems. We presented a smart energy efficient single overhead based wide angle lens camera models. The presented models are smart in term of surveillance by covering the large field of view and solving the problem of occlusion. Furthermore, the single overhead based camera model is also used as a centralized camera to make a hybrid surveillance system by controlling frontal based cameras. It also solves the privacy issues in the different real-life environment. We have analyzed the installation cost and energy usage of multiple camera solutions and come up with the solution that installing an overhead camera will able to solve the above-discussed issues. In this paper we opened a new direction in which activity, suspicious motion, unusual activities, behaviours gait analysis etc can be done using overhead view in contrast to the frontal view. All type of techniques or methods that are developed in the frontal view can also be considered in overhead view. As all the time we don't need facial images, in such scenario we can also use the hybrid view model. Instead of camera calibration, we can use a hybrid model consisting of one overhead camera and required number of frontal cameras. We can also use the overhead camera as a motion detection. It can also be used as an alerting signal for other frontal cameras. In short, we can use the overhead camera system as a facilitating system for a frontal view.

This paper sheds more light on the smart surveillance system solutions. In future, we will extend this work with smart sensor based surveillance system. We will also intend to implement the discussed models in future surveillance systems. We also use the discussed models for 3D modelling, image stitching, camera calibration and more ground-breaking research ideas.

\section{ACKNOWLEDGMENT}

We are thankful to the Institute of Management Sciences and Higher Education Commission (HEC) Pakistan, funding for this research work via NRPU project under project number 5840/KPK/NRPU/RND/HEC/2016.

\section{REFERENCES}

[1] UN, 2019 (accessed March 3, 2019), https://www.un.org/development/desa/en/news/population/2018revision-of-world-urbanization-prospects.html/.

[2] M. S. K. Mishra, F. JTMCOE, and K. Bhagat, "A survey on human motion detection and surveillance," International Journal of Advanced Research in Electronics and Communication Engineering (IJARECE) Volume, vol. 4, 2015.

[3] K. Mikolajczyk, C. Schmid, and A. Zisserman, "Human detection based on a probabilistic assembly of robust part detectors," in European Conference on Computer Vision. Springer, 2004, pp. 69-82.

[4] J.-S. Yeh, C.-C. Chang, T.-L. Chia, S.-Y. Chiang, and P.-S. Huang, "Cooperative dual camera surveillance system for real-time object searching and close-up viewing," in Intelligent Green Building and Smart Grid (IGBSG), 2016 2nd International Conference on. IEEE, 2016, pp. 1-5.

[5] P. Natarajan, P. K. Atrey, and M. Kankanhalli, "Multi-camera coordination and control in surveillance systems: A survey," ACM Transactions on Multimedia Computing, Communications, and Applications (TOMM), vol. 11, no. 4, p. 57, 2015.

[6] A. Bialkowski, S. Denman, S. Sridharan, C. Fookes, and P. Lucey, "A database for person re-identification in multi-camera surveillance networks," in Digital Image Computing Techniques and Applications (DICTA), 2012 International Conference on. IEEE, 2012, pp. 1-8.

[7] J.-L. Chua, Y. C. Chang, and W. K. Lim, "A simple vision-based fall detection technique for indoor video surveillance," Signal, Image and Video Processing, vol. 9, no. 3, pp. 623-633, 2015.

[8] I. Cohen, A. Garg, and T. S. Huang, "Vision-based overhead view person recognition," in Pattern Recognition, 2000. Proceedings. 15th International Conference on, vol. 1. IEEE, 2000, pp. 1119-1124.

[9] I. Ahmed and J. N. Carter, "A robust person detector for overhead views," in Pattern Recognition (ICPR), 2012 21st International Conference on. IEEE, 2012, pp. 1483-1486.

[10] I. Ahmed and A. Adnan, "A robust algorithm for detecting people in overhead views," Cluster Computing, pp. 1-22, 2017.

[11] N. Dalal and B. Triggs, "Histograms of oriented gradients for human detection," in Computer Vision and Pattern Recognition, 2005. CVPR 2005. IEEE Computer Society Conference on, vol. 1. IEEE, 2005, pp. 886-893.

[12] R. Nakatani, D. Kouno, K. Shimada, and T. Endo, "A person identification method using a top-view head image from an overhead camera." JACIII, vol. 16, no. 6, pp. 696-703, 2012.

[13] L. Del Pizzo, P. Foggia, A. Greco, G. Percannella, and M. Vento, "A versatile and effective method for counting people on either rgb or depth overhead cameras," in Multimedia \& Expo Workshops (ICMEW), 2015 IEEE International Conference on. IEEE, 2015, pp. 1-6. 
[14] J. García, A. Gardel, I. Bravo, J. L. Lázaro, M. Martínez, and D. Rodríguez, "Directional people counter based on head tracking," IEEE Transactions on Industrial Electronics, vol. 60, no. 9, pp. 39914000, 2013.

[15] T.-W. Choi, D.-H. Kim, and K.-H. Kim, "Human detection in top-view depth image," Contemporary Engineering Sciences, vol. 9, no. 11, pp. 547-552, 2016.

[16] P. Blondel, A. Potelle, C. Pégard, and R. Lozano, "Human detection in uncluttered environments: From ground to uav view," in Control Automation Robotics \& Vision (ICARCV), 2014 13th International Conference on. IEEE, 2014, pp. 76-81.

[17] D. de Oliveira and M. Wehrmeister, "Using deep learning and low-cost $\mathrm{rgb}$ and thermal cameras to detect pedestrians in aerial images captured by multirotor uav," Sensors, vol. 18, no. 7, p. 2244, 2018.

[18] Y. Jiang and J. Ma, "Combination features and models for human detection," in Proceedings of the IEEE Conference on Computer Vision and Pattern Recognition, 2015, pp. 240-248.

[19] V. Carletti, L. Del Pizzo, G. Percannella, and M. Vento, "An efficient and effective method for people detection from top-view depth cameras," in Advanced Video and Signal Based Surveillance (AVSS), 2017 14th IEEE International Conference on. IEEE, 2017, pp. 1-6.

[20] Z. Q. Al-Zaydi, D. L. Ndzi, M. L. Kamarudin, A. Zakaria, and A. Y.
Shakaff, "A robust multimedia surveillance system for people counting," Multimedia Tools and Applications, vol. 76, no. 22, pp. 23 777-23 804, 2017.

[21] A.-S. Liu, T.-W. Hsu, P.-H. Hsiao, Y.-C. Liu, and L.-C. Fu, "The manhunt network: People tracking in hybrid-overlapping under the vertical top-view depth camera networks," in Advanced Robotics and Intelligent Systems (ARIS), 2016 International Conference on. IEEE, 2016, pp. 1-6.

[22] A. Burbano, S. Bouaziz, and M. Vasiliu, "3d-sensing distributed embedded system for people tracking and counting," in 2015 International Conference on Computational Science and Computational Intelligence (CSCI). IEEE, 2015, pp. 470-475.

[23] T.-E. Tseng, A.-S. Liu, P.-H. Hsiao, C.-M. Huang, and L.-C. Fu, "Real-time people detection and tracking for indoor surveillance using multiple top-view depth cameras," in 2014 IEEE/RSJ International Conference on Intelligent Robots and Systems. IEEE, 2014, pp. 40774082 .

[24] S. Tang, B. Andres, M. Andriluka, and B. Schiele, "Multi-person tracking by multicut and deep matching," in European Conference on Computer Vision. Springer, 2016, pp. 100-111.

[25] Reolink, 2019 (accessed February 3, 2019), https://reolink.com/cctv-ipsecurity-camera-power-consumption/. 